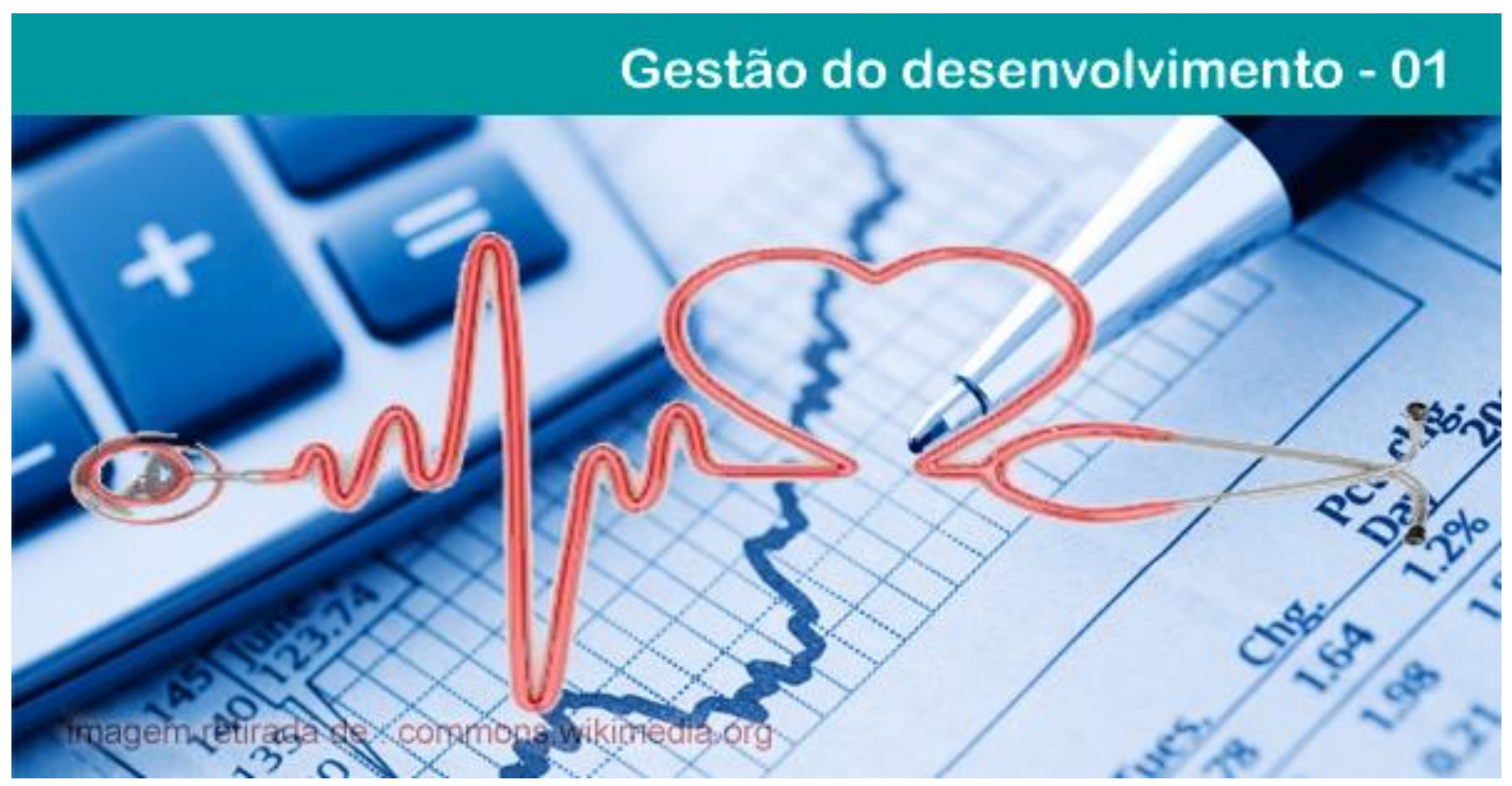

\title{
O IMPACTO DA ATIVIDADE ECONÔMICA SOBRE A SAÚDE DOS INDIVÍDUOS: UMA ANÁLISE A PARTIR DA PNAD 2008.
}

Pablo Aurélio Lacerda de Almeida Pinto

Professor da Universidade de Pernambuco (UPE). Doutor em Economia pela Universidade Federal de Pernambuco (PIMES/UFPE). Mestre em Economia pela Universidade Federal da Paraíba (UFPB). Graduado em Ciências Econômicas pela Universidade Federal de Campina Grande (UFCG). Graduado em Administração pela Universidade Estadual da Paraíba (UEPB). E-mail: pabloaurelioap@ @otmail.com.

\section{Edivaldo Constantino das Neves Júnior}

Doutorando em Economia do Desenvolvimento, Instituto de Pesquisas Econômicas/Universidade de São Paulo (USP/SP). Mestre em Teoria Econômica pela Universidade Federal de Pernambuco (UFPE). Bacharel em Ciências Econômicas pela UFPE. Integrante do Núcleo de Economia Urbana e Regional da USP (NEREUS/USP).E-mail: ecnjr07@yahoo.com.br.

\section{Danilo Arruda}

Doutor em Economia pela Universidade Federal do Rio de Janeiro (UFRJ). Mestre em Economia pela Universidade Federal da Paraíba. Graduado em Economia pela Universidade Federal de Campina Grande. Pesquisador da Rede de Pesquisa em Sistemas e Arranjos Produtivos e Inovativos Locais RedeSist. E-mail: dr_arruda@yahoo.com.br.

\section{Elaine Peixoto Borin}

Professora Adjunta da Faculdade de Engenharia da Universidade do Estado do Rio de Janeiro (UERJ). Doutora em Planejamento Urbano e Regional pela Universidade Federal do Rio de Janeiro (UFRJ). Mestre em Engenharia de Produção pela Universidade Federal Fluminense (UFF). Graduada em Engenharia Civil pela UFRJ. Pesquisadora da Rede de Pesquisa em Sistemas e Arranjos Produtivos e Inovativos Locais RedeSist/UFRJ. E-mail: elaine.borin@ig.com.br.

Resumo: Este trabalho tem como objetivo realizar uma análise das condições gerais de saúde da população do Estado de Pernambuco, com um olhar no impacto dos setores de atividades econômica em relação às doenças crônicas e aspectos da saúde dos indivíduos. Pretende-se, assim, subsidiar decisões voltadas para a adoção de medidas de prevenção, além de ajudar na discussão pública sobre o impacto destes agravos no crescimento

\section{POLÊM!CA $\mid$ LABORE (}

Polêmica - Revista Eletrônica da Uerj - Rua São Francisco Xavier, 524, $1^{\circ}$ andar

bloco D, sl.1001 • Tels.: +55 21 2334-4088/4087 • http://www.e-publicacoes.uerj.br/index.php/polemica/index http://www.labore.uerj.br • laboreuerj@yahoo.com.br 
econômico. Os dados foram extraídos da Pesquisa Nacional de Amostragem Domiciliar (PNAD) de 2008, e a partir de então foi estimado um modelo probit, utilizado para variável dependente discreta (assume o valor zero ou um), em que a variável dependente é representada pelas doenças crônicas e as variáveis independentes representam as informações das características individuais. Como resultado, encontrou-se que, no que se refere à probabilidade do indivíduo possuir determinada doença crônica dentre as 12 (doze) doenças listadas na PNAD, apenas três variáveis, que captam o efeito do setor de atividade, mostraram-se significantes estatisticamente. São elas: doença de coluna ou costas, hipertensão e tuberculose. Além disso, diferenças de gênero e escolaridade apresentaram relativo grau de importância.

Palavras-chaves: Saúde. Setores de Atividades Econômicas. Doenças Crônicas.

\title{
ACTIVITY IMPACT OF ECONOMIC HEALTH OF INDIVIDUALS: AN ANALYSIS FROM 2008 PNAD
}

\begin{abstract}
This paper aims to make an analysis of the general conditions of health of the State of Pernambuco's population, with detachment on the impact sectors of the economy have over chronical diseases and individuals' health aspects. Therefore, this paper intend to subsidize the decisions to the adoption of prevention's measure, and to support the public discussionabout the impact of these barriers to the economic development. The datas used were from the Pesquisa Nacional de Amostragem Dominiciliar (PNAD) in 2008, and based on them a probit model was estimated, used for discrete dependent variable (takes the value zero or one), in which the dependent variable is represented by the chronical diseases and the independent variables have the information of individual characteristics. As result, we find that, in matters of the individual's probability to has a specific chronical disease among the 12 (twelve) listed disease in the PNAD, only three variables that capture the effect of the activity sector appeared statistically significant. Besides that, differences in the gender and scholarship showed a relatively importance grade.
\end{abstract}

Keywords: Health. Sectors of the Economy. Chronical Diseases.

\section{Introdução}

De acordo com dados da Organização Internacional do Trabalho (OIT), cerca de 2,2 milhões de trabalhadores morrem todos os anos devido a acidentes ou doenças relacionadas ao trabalho (DRT) no mundo todo (OIT, 2005). Como consequência, o número de estudos sobre os impactos negativos que as mudanças estruturais ocorridas no mundo do trabalho exercem na saúde dos trabalhadores aumentou (LACAZ, 2000).

Wunsch Filho (2004) argumenta que se faz necessário considerar a essência das mudanças sociais e econômicas ocorridas no mundo globalizado para avaliar seus resultados na saúde da população trabalhadora. Minayo-Gomes e Lacaz (2005) adicionam as condições socioeconômicas, tecnológicas e organizacionais para explicar os agravos à saúde do trabalhador.

Mendes (2005) criou três categorias de doenças segundo sua relação com o trabalho: o grupo I categoriza o trabalho como causa necessária; no grupo II o trabalho se apresenta como fator contributivo, mas não necessário para o aparecimento de doenças; e o grupo III, das doenças relacionadas ao trabalho, considera o trabalho como provocador de um distúrbio latente ou agravador de doença já estabelecida. A partir de 2001, o Ministério da Saúde (MS)

\section{POLÊM!CA $\mid$ LABORE}

Polêmica - Revista Eletrônica da Uerj - Rua São Francisco Xavier, 524, $1^{\circ}$ andar

bloco D, sl.1001 • Tels.: +55 21 2334-4088/4087 • http://www.e-publicacoes.uerj.br/index.php/polemica/index

http://www.labore.uerj.br • laboreuerj@yahoo.com.br 
adotou a classificação proposta por Schilling (1984) das doenças relacionadas ao trabalho no âmbito do Sistema Único de Saúde (SUS).

A lista das doenças relacionadas ao trabalho, como ficou conhecida, foi padronizada pela Classificação Internacional das Doenças, em sua décima revisão (CID-10) (Ministério da Saúde, 2001). No Brasil, o registro de DRT aumentou de 5.025, em 1988, para 30.334, em 2005, entre os trabalhadores do Regime Geral da Previdência Social ${ }^{1}$. O contrário ocorreu em países desenvolvidos, onde houve diminuição na ocorrência destas doenças. Nos Estados Unidos, o Bureau of Labor Statistics (2002) registrou 294.500 registros de DRT que declinou em 2005 para 242.500 notificações $^{2}$. As causas apontadas para o declínio das DRT são diversas, desde o sub-registro a fatores macroeconômicos. Outro fator importante poderia ser a prevenção primária nos locais de trabalho.

Segundo Souza et al. (2008), os trabalhadores do Regime Geral da Previdência Social e que podem receber benefícios por DRT são: empregados, trabalhadores avulsos e segurados especiais. A responsabilidade pela atribuição do nexo causal entre as enfermidades e o trabalho é dos médicos peritos do Instituto Nacional do Seguro Social (INSS), uma autarquia ligada ao Ministério da Previdência Social (MPS).

O estudo da distribuição das DRT, entre os diversos ramos de atividades econômicas, pode subsidiar decisões voltadas para a adoção de medidas de prevenção, além de ajudar na discussão pública sobre o impacto destes agravos no crescimento econômico. Segundo Godoy et al. (2007), as implicações econômicas das doenças relacionadas à atividade laboral são importantes, pois elas irão influenciar, de modo marcante e decisivo, o comportamento dos agentes econômicos no mercado de trabalho, estando associada à redução nas horas de trabalho, a menores taxas de salário, à aposentadoria precoce, à saída antecipada do mercado de trabalho e a programas de transferências de renda. Além disso, as doenças relacionadas ao trabalho não afetam apenas o indivíduo, mas também toda a sua família.

Avaliar o impacto das DRT e a perda de capacidade funcional e o afastamento da força de trabalho, no Brasil, é imprescindível pela carência de informações, que são escassas e subnumeradas. O presente trabalho visa contribuir metodologicamente no aprimoramento da relação de causalidade entre doenças crônicas e condições socioeconômicas dos indivíduos, procurando também subsidiar políticas públicas de saúde do trabalhador.

\footnotetext{
${ }^{1}$ Ministério da Previdência Social. Anuário Estatístico da Previdência Social.

${ }^{2}$ Bureau of Labor Statistics. Washington: United States Department of Labor, 2006.
}

\section{POLÊM!CA $\mid$ LABORE}


O objetivo deste trabalho é realizar uma análise probabilística (através do modelo probit) dos impactos relativos às condições socioeconômicas do indivíduo sobre a saúde do trabalhador, destacando-se relações entre doenças, atividade econômica e fatores socioeconômicos. Serão utilizadas as informações extraídas da Pesquisa Nacional de Amostra Domiciliar (PNAD) para o ano de 2008.

O presente artigo está dividido em mais seis seções além desta introdução. A próxima seção apresenta uma revisão da literatura sobre o tema das doenças relacionadas à atividade econômica. Na terceira seção, apresenta-se a metodologia utilizada para implementar a estratégia empírica, contendo toda a estrutura econométrica A quarta seção contém informações sobre os bancos de dados utilizados e a análise descritiva dos mesmos. A quinta seção de análise de resultados, apresenta as estimações realizadas e a discussão dos resultados. Por fim, as considerações finais.

\section{Revisão da literatura}

Vários estudos têm incorporado causas não biológicas para interpretação do processo saúde-doença. Burnley (1998) procurou examinar as desigualdades na incidência de doenças isquêmicas do coração, na Austrália, entre 1969 e 1994. O autor verificou que, embora se observasse uma queda nas taxas de mortalidade, as diferenças entre as "ocupações profissionais" aumentavam, ou seja, o setor de atividade onde o indivíduo trabalha.

Para Kaplan (1996) existe um substancial corpo de evidências que demonstram a forte associação inversa entre as classes sociais e os efeitos sobre a saúde. Contudo, o autor chama a atenção de que o foco de análise deve considerar as características da comunidade em que as pessoas vivem, ao invés dos atributos individuais que indicam as condições socioeconômicas.

Em estudo realizado com dados de 2.610 homens franceses, entre 50 a 60 anos, Michaux et al (1996) encontraram diferenças significativas entre as condições socioeconômicas e os fatores de risco coronariano. Considerando as categorias profissionais (CSP2 - comerciantes e chefes de empresas; CSP3 - professores e intelectuais; CSP4 profissões intermediárias; CSP5 - empregados; e, CSP6 - operários), foi possível encontrar que o tabagismo, o colesterol total, a pressão arterial diastólica e o diabetes não apresentaram diferenças entre as categorias profissionais.

\section{POLÊM!CA $\mid$ LABORE}


Rosengren et al (1998), a partir dos dados de saúde dos trabalhadores suecos, identificaram que a mortalidade é três vezes maior nos trabalhadores não qualificados do que nos gerentes e altos funcionários. Foram verificadas, ainda, que as baixas classes ocupacionais estão associadas com a alta prevalência do fumo, baixa integração social, baixo nível de atividades em casa e fora de casa, baixo suporte emocional, bem como uma baixa capacidade de percepção da própria saúde. Todavia, não foi encontrada associação das classes ocupacionais mais baixas com a elevação da pressão arterial, dos triglicerídeos e do colesterol.

Impacto dos fatores macroeconômicos na saúde pública é alvo de discussão para Kaplan e Lynch (1999); estes autores comentam a respeito de como os aspectos sociais e econômicos podem ter alguma influência sobre o estado de saúde da sociedade, bem como sobre a prevenção primária das doenças. O enredo de globalização da economia, que provoca alta competitividade, desemprego, insegurança no trabalho, desigualdades sociais, baixa coesão social etc, poderia estar provocando uma elevada pressão sobre os trabalhadores e, por isso, favorecendo o aumento do estresse, da pressão arterial e das doenças cardiovasculares. Por outro lado, o trabalhador estaria com seu tempo diminuído para cuidar de si próprio, além do maciço programa de marketing desenvolvido para vender cigarros, alimentos do tipo "fast food" e/ou refrigerantes, os quais, notadamente, favorecem uma pior condição de saúde.

\section{Metodologia}

Com o intuito de analisar as condições gerais de saúde da população pernambucana, focaremos a investigação no impacto que os setores de atividades possam causar na probabilidade do indivíduo possuir ou não uma série de doenças crônicas. Utilizaremos um modelo probabilístico, cuja variável dependente será representada pelas doenças crônicas e as variáveis explicativas serão denotadas por características individuais. Como estamos tratando de variáveis dependentes discretas, o modelo permite estimar a influência das variáveis explicativas na tomada de decisão binária, do tipo 'ou uma ou outra'.

Será aplicado o modelo probabilístico probit, que consegue relacionar fatores explicativos à probabilidade da "escolha", empregando a função de distribuição de probabilidade acumulada de uma variável aleatória normal padrão, de modo que a probabilidade permaneça entre [0,1]. Assim, podemos analisar a decisão a ser observada no

\section{POLÊM!CA $\mid$ LABORE}


nosso estudo de acordo com a avaliação das características consideradas relevantes, bem como verificar os seus efeitos marginais, ou seja, a variação na probabilidade de 'escolha', caso haja variação em uma unidade de alguma das variáveis explicativas, todas as outras variáveis permanecendo constantes. Devido ao fato de se tratar de um modelo estatístico não linear, o melhor método de estimação é por máxima verossimilhança. O modelo toma a seguinte estrutura:

$A_{i}^{*}=S E X_{i} \sigma+I D A_{i} \delta+\operatorname{COR}_{i} \varphi+\operatorname{STC}_{i} \beta+E S C_{i} \gamma+\operatorname{REN}_{i} \alpha+F U M_{i} \phi+A T V_{i} \theta+\operatorname{SAT}_{i} v+\mu_{i}$
$A_{i}= \begin{cases}1 & \text { se } \mu_{i} \geq-S E X_{i} \sigma-I D A_{i} \delta-\operatorname{COR}_{i} \varphi-S T C_{i} \beta-E S C_{i} \gamma-\operatorname{REN}_{i} \alpha-F U M_{i} \phi-A T V_{i} \theta-S A T_{i} v \\ 0 & \text { caso contrário }\end{cases}$

Assumimos, então, que $A_{i}$ na equação representa o fato do indivíduo $i$ possuir ou não determinada doença crônica. Em relação às variáveis explicativas, temos que, para o indivíduo $i: S E X_{i}$ representa o sexo, $I D A_{i}$ representa a idade, $C O R_{\mathrm{i}}$ indica a cor, $S T C_{i}$ indica a situação censitária, $E S C_{i}$ representa a escolaridade, $R E N_{\mathrm{i}}$ a renda de todas as fontes e $F U M_{\mathrm{i}} \mathrm{o}$ fato do indivíduo estar ou não fumando, $A T V_{i}$ indica atividades físicas e $S A T_{\mathrm{i}}$ é uma dummy que indica o setor de atividade que o indivíduo está inserido.

\section{Análise descritiva dos dados}

Os dados foram extraídos da PNAD 2008, cuja edição contou com um suplemento especial de saúde. Vale destacar que a contemplação do tema saúde nos suplementos da PNAD possui uma regularidade de 5 anos, sendo padronizado a partir do ano de 1998. Assim, tem-se dados que avaliam a saúde dos brasileiros nos anos de 1998, 2003 e 2008, sendo este último ano o foco de nossa análise. As variáveis de saúde foram separadas em conjuntos para otimizar a nossa investigação, a saber: incidência de doenças por setores de atividades econômicas e controlando por decis de renda (Tabela 1) e caracterização geral de saúde por setores de atividades econômicas e também atentando para os decis de renda (Tabela 2).

O objetivo é proceder uma análise das condições gerais de saúde da população do Estado de Pernambuco, com um olhar no impacto dos setores de atividades em relação às doenças crônicas e aspectos da saúde dos indivíduos. Para definição de setores de atividades, procedeu-se com a utilização da variável que define os grupamentos de atividade principal do empreendimento do trabalho principal da semana de referência para pessoas de 10 anos ou

\section{POLÊM!CA $\mid$ LABORE}


mais de idade. Entre os grupamentos que constam na PNAD tem-se agrícola, outras atividades industriais, indústria de transformação, construção, comércio e reparação, alojamento e alimentação, transporte, armazenagem e comunicação, administração pública, educação, saúde e serviços sociais, serviços domésticos, outros serviços coletivos, sociais e pessoais e por fim, outras atividades. Assim, reordenamos essa classificação em setores agrícola, indústria e comércio e serviços.

Utilizou-se a renda de todas as fontes, variáveis de doenças crônicas estabelecidas no banco da PNAD e também variáveis de características sociodemográficas (ou econômicas), como sexo, idade, anos de escolaridade, setor censitário, entre outras, para construir os resultados. Em relação às doenças crônicas, é importante destacar que a pergunta na PNAD é: “Algum médico ou profissional de saúde disse que tem doença de coluna ou costas?”. Essa forma de questionar, para todas as doenças, também inclui a questão do acesso à saúde na determinação da doença do indivíduo. Pode ser que pessoas de uma renda maior tenham mais acesso aos serviços de saúde e por isso declarem ter uma maior série de doenças. Por isso, é essencial controlar pela renda dos indivíduos, para capturar um efeito mais puro das variáveis de doenças, evitando problemas de interpretação incorreta da realidade.

Assim sendo, iremos proceder uma análise dos resultados descritivos obtidos nessa primeira etapa de diagnosticar o panorama da saúde brasileira atentando para os setores de atividades econômicas. De acordo com a Tabela 1, percebe-se que existem doenças que possuem uma maior prevalência nos decis mais baixos da população, enquanto outras possuem uma maior prevalência nos decis mais elevados. Existe ainda a situação na qual não é possível determinar uma tendência clara de doenças em relação aos decis de renda, muito embora se note uma relação não linear. Nessa linha de argumentação, nota-se que ter doença de coluna ou costas, além de hipertensão, apresentaram maior prevalência na população pernambucana, independente do setor de atividade que se leve em consideração.

Entre as aferições que não apresentaram uma tendência de crescimento ou decrescimento em relação à renda (existe uma relação não linear) tem-se doença de coluna ou costas, câncer, tuberculose, cirrose, artrite e reumatismo (apenas para agrícola e indústria), bronquite (apenas agrícola e comércio e serviços) e tendinite ou tenossinovite (apenas para agrícola e indústria). Já doenças como hipertensão, diabetes, doença do coração, tendinite (comércio e serviços) e depressão (agrícola) apresentam uma maior proporção de indivíduos

\section{POLÊM!CA $\mid$ LABORE}


doentes em faixas de renda mais elevadas. Por fim, em artrite ou reumatismo (comércio e serviços), bronquite (indústria), doença renal crônica, depressão (indústria e comércio e serviços) verifica-se uma tendência decrescente, à medida que se caminha da base para topo da distribuição de renda.

Tabela - 1: Doenças controladas por decis de renda e setores de atividades

\begin{tabular}{|c|c|c|c|c|c|c|c|c|c|c|c|}
\hline & \multicolumn{11}{|c|}{ Decis de Renda } \\
\hline & 1 & 2 & 3 & 4 & 5 & 6 & 7 & 8 & 9 & 10 & Total \\
\hline \multicolumn{12}{|l|}{ Agricultura } \\
\hline $\begin{array}{l}\text { Tem doença de } \\
\text { Coluna ou Costas }\end{array}$ & 20.20 & 16.72 & 18.17 & 19.71 & 21.26 & 24.73 & 21.62 & 22.43 & 41.82 & 21.45 & 21.43 \\
\hline $\begin{array}{l}\text { Tem Artrite ou } \\
\text { Reumatismo }\end{array}$ & 7.42 & 5.76 & 2.37 & 5.06 & 12.02 & 15.05 & 10.09 & 7.68 & 16.48 & 2.55 & 8.20 \\
\hline Tem Câncer & 0.00 & 0.17 & 0.00 & 0.93 & 0.00 & 1.08 & 0.72 & 0.00 & 1.33 & 0.00 & 0.39 \\
\hline Tem Diabetes & 3.13 & 2.97 & 1.96 & 0.93 & 3.35 & 5.91 & 2.16 & 3.54 & 6.63 & 7.64 & 3.40 \\
\hline $\begin{array}{l}\text { Tem Bronquite ou } \\
\text { Asma }\end{array}$ & 3.22 & 2.00 & 1.68 & 3.74 & 2.37 & 3.76 & 1.44 & 3.54 & 1.33 & 2.55 & 2.55 \\
\hline Tem Hipertensão & 17.29 & 11.93 & 11.22 & 12.23 & 24.20 & 27.42 & 18.20 & 19.76 & 33.51 & 34.18 & 18.53 \\
\hline $\begin{array}{l}\text { Tem Doença do } \\
\text { Coração }\end{array}$ & 2.91 & 0.40 & 0.98 & 6.54 & 1.96 & 5.38 & 2.88 & 3.10 & 4.54 & 5.10 & 2.88 \\
\hline $\begin{array}{l}\text { Tem Doença Renal } \\
\text { Crônica }\end{array}$ & 1.07 & 1.60 & 0.98 & 1.87 & 3.92 & 0.54 & 1.44 & 1.47 & 0.00 & 0.00 & 1.33 \\
\hline Tem Depressão & 3.89 & 5.20 & 3.85 & 2.80 & 2.94 & 1.08 & 2.16 & 2.50 & 6.64 & 7.64 & 3.57 \\
\hline Tem Tuberculose & 0.22 & 0.40 & 0.00 & 0.93 & 0.00 & 0.54 & 0.00 & 0.00 & 0.00 & 0.00 & 0.25 \\
\hline $\begin{array}{l}\text { Tem Tendinite ou } \\
\text { Tenossinovite }\end{array}$ & 0.00 & 0.00 & 0.00 & 0.93 & 1.96 & 0.54 & 0.72 & 2.07 & 1.88 & 0.00 & 0.61 \\
\hline Tem Cirrose & 0.00 & 0.00 & 0.00 & 1.87 & 0.98 & 0.54 & 0.00 & 0.00 & 0.00 & 0.00 & 0.29 \\
\hline \multicolumn{12}{|l|}{ Indústria } \\
\hline $\begin{array}{l}\text { Tem doença de } \\
\text { Coluna ou Costas }\end{array}$ & 10.33 & 24.00 & 12.27 & 14.74 & 15.53 & 13.67 & 15.95 & 14.04 & 19.87 & 12.36 & 15.12 \\
\hline $\begin{array}{l}\text { Tem Artrite ou } \\
\text { Reumatismo }\end{array}$ & 4.13 & 0.90 & 3.62 & 5.95 & 2.72 & 0.00 & 3.76 & 1.88 & 3.99 & 3.49 & 3.28 \\
\hline Tem Câncer & 0.00 & 0.00 & 0.00 & 0.00 & 0.00 & 0.00 & 0.00 & 0.00 & 0.00 & 0.47 & 0.04 \\
\hline Tem Diabetes & 1.03 & 0.00 & 1.72 & 2.27 & 1.71 & 0.00 & 0.62 & 4.46 & 2.03 & 3.96 & 2.13 \\
\hline $\begin{array}{l}\text { Tem Bronquite ou } \\
\text { Asma }\end{array}$ & 8.04 & 9.56 & 1.72 & 2.70 & 1.52 & 4.56 & 4.07 & 2.39 & 3.43 & 3.78 & 3.41 \\
\hline Tem Hipertensão & 11.13 & 14.44 & 8.83 & 13.94 & 7.78 & 7.59 & 14.48 & 9.96 & 13.71 & 21.22 & 12.26 \\
\hline $\begin{array}{l}\text { Tem Doença do } \\
\text { Coração }\end{array}$ & 1.04 & 0.90 & 1.60 & 1.84 & 1.01 & 3.04 & 2.90 & 0.94 & 2.03 & 1.60 & 1.73 \\
\hline $\begin{array}{l}\text { Tem Doença Renal } \\
\text { Crônica }\end{array}$ & 2.47 & 0.00 & 0.00 & 1.04 & 0.00 & 1.52 & 1.78 & 1.60 & 0.00 & 1.13 & 0.93 \\
\hline Tem Depressão & 6.60 & 4.33 & 2.13 & 3.13 & 1.01 & 1.52 & 3.02 & 2.96 & 1.71 & 1.89 & 2.59 \\
\hline
\end{tabular}

\section{POLÊM!CA $\mid$ LABORE}

Polêmica - Revista Eletrônica da Uerj - Rua São Francisco Xavier, 524, $1^{\circ}$ andar bloco D, sl.1001 • Tels.: +55 21 2334-4088/4087 • http://www.e-publicacoes.uerj.br/index.php/polemica/index http://www.labore.uerj.br • laboreuerj@yahoo.com.br 


\begin{tabular}{|c|c|c|c|c|c|c|c|c|c|c|c|}
\hline Tem Tuberculose & 0.00 & 0.00 & 0.00 & 0.00 & 1.21 & 0.00 & 0.00 & 0.24 & 0.32 & 0.00 & 0.18 \\
\hline $\begin{array}{l}\text { Tem Tendinite ou } \\
\text { Tenossinovite }\end{array}$ & 4.53 & 2.17 & 1.19 & 1.78 & 1.01 & 0.00 & 2.16 & 1.17 & 2.67 & 3.30 & 1.84 \\
\hline Tem Cirrose & 0.00 & 0.00 & 0.00 & 0.00 & 0.00 & 0.00 & 0.00 & 0.00 & 0.00 & 0.94 & 0.08 \\
\hline \multicolumn{12}{|l|}{ Comércio e Serviços } \\
\hline $\begin{array}{l}\text { Tem doença de } \\
\text { Coluna ou Costas }\end{array}$ & 12.75 & 13.19 & 15.62 & 12.68 & 12.16 & 15.17 & 13.17 & 13.75 & 11.82 & 15.15 & 13.60 \\
\hline $\begin{array}{l}\text { Tem Artrite ou } \\
\text { Reumatismo }\end{array}$ & 6.06 & 6.07 & 4.96 & 2.89 & 2.99 & 5.67 & 3.56 & 4.36 & 5.09 & 3.90 & 4.33 \\
\hline Tem Câncer & 0.00 & 0.45 & 0.00 & 0.10 & 0.14 & 0.00 & 0.21 & 0.38 & 0.18 & 0.55 & 0.23 \\
\hline Tem Diabetes & 2.43 & 1.77 & 3.50 & 1.98 & 1.89 & 3.09 & 3.64 & 2.40 & 4.10 & 5.21 & 3.23 \\
\hline $\begin{array}{l}\text { Tem Bronquite ou } \\
\text { Asma }\end{array}$ & 3.55 & 5.24 & 4.03 & 2.87 & 2.56 & 1.55 & 1.83 & 3.92 & 3.20 & 3.07 & 3.16 \\
\hline Tem Hipertensão & 11.90 & 14.36 & 14.93 & 13.36 & 12.65 & 12.59 & 14.28 & 16.14 & 19.23 & 19.27 & 15.52 \\
\hline $\begin{array}{l}\text { Tem Doença do } \\
\text { Coraçãoo }\end{array}$ & 1.80 & 2.34 & 2.07 & 2.50 & 2.22 & 4.13 & 3.74 & 2.79 & 3.80 & 3.74 & 3.02 \\
\hline $\begin{array}{l}\text { Tem Doença Renal } \\
\text { Crônica }\end{array}$ & 1.53 & 1.40 & 1.35 & 1.10 & 0.96 & 2.06 & 0.64 & 0.73 & 0.94 & 0.58 & 1.00 \\
\hline Tem Depressão & 3.46 & 5.35 & 3.74 & 4.44 & 1.94 & 2.58 & 3.81 & 3.55 & 1.98 & 3.28 & 3.39 \\
\hline Tem Tuberculose & 0.00 & 0.57 & 0.20 & 0.41 & 0.14 & 0.00 & 0.26 & 0.00 & 0.09 & 0.18 & 0.18 \\
\hline $\begin{array}{l}\text { Tem Tendinite ou } \\
\text { Tenossinovite }\end{array}$ & 2.56 & 1.89 & 2.72 & 2.08 & 3.02 & 1.55 & 3.51 & 3.81 & 3.37 & 5.23 & 3.26 \\
\hline Tem Cirrose & 0.00 & 0.00 & 0.10 & 0.00 & 0.34 & 0.00 & 0.00 & 0.21 & 0.09 & 0.15 & 0.10 \\
\hline
\end{tabular}

Fonte: PNAD (2008), elaboração própria.

É importante discutir alguns desses resultados. Pessoas que trabalham no setor agrícola apresentam uma maior prevalência de doenças quando se compara com os outros dois setores. Porém, quando é focada a tendinite, percebe-se que no setor agrícola quase não existe prevalência dessa enfermidade, enquanto que no setor de comércio e serviços cerca de 3,2\% dos pernambucanos declaram sofrer da mesma. Essas informações evidenciam uma relação direta entre o tipo de esforço físico ou mental desenvolvido em cada setor de atividade e as possíveis enfermidades que as pessoas podem sofrer. Destaca-se ainda que, a prevalência de doença nas costas é maior no setor agrícola, mas não se verificou uma tendência clara com os decis de renda. O fato é que o trabalho realizado nesse setor exige uma boa condição física das pessoas.

Além disso, percebe-se que a hipertensão atinge uma maior proporção de pessoas nos decis de renda mais altos, qualquer que seja o setor de atividade. Isso pode estar relacionado a questões como o estresse, já que pessoas de maior poder aquisitivo são responsáveis por decisões de grande porte e, assim, acabam ficando expostas a uma maior quantidade de

\section{POLÊM!CA | LABORẸ}

Polêmica - Revista Eletrônica da Uerj - Rua São Francisco Xavier, 524, $1^{\circ}$ andar

bloco D, sl.1001 • Tels.: +55 21 2334-4088/4087 • http://www.e-publicacoes.uerj.br/index.php/polemica/index

http://www.labore.uerj.br • laboreuerj@yahoo.com.br 
problemas que provocam o estresse e que acarretam problemas de pressão arterial. Note ainda que a diabetes também apresenta o mesmo comportamento.

A Tabela 2 mostra algumas características da saúde da população pernambucana tendo como foco de análise os decis de renda e os setores de atividades. Percebe-se que a proporção de pessoas que declaram ter estado de saúde ótimo ou bom, apresenta uma tendência crescente ao longo da distribuição de renda, independentemente do setor em análise. Pessoas de renda mais elevada possuem um melhor estado de saúde. A literatura discute que a autoavaliação do estado de saúde busca mensurá-lo utilizando um critério subjetivo, uma vez que depende do julgamento do indivíduo acerca do seu próprio estado. Assim o indivíduo não apenas responde com base na simples presença ou não de uma doença, mas procura ponderar a sua resposta a características que extrapolam o significado de saúde.

Quando analisa-se a proporção de pessoas que consultaram o médico nos últimos 12 meses, observa-se uma tendência crescente ao longo dos decis de renda nos setores agrícola e de comércio e serviços. Uma suposta interpretação para não constatação dessa evidência no setor industrial é que a grande quantidade de consulta ao médico significa perda de dias de trabalho e baixa produtividade, o que obviamente não é bom para o empregador. Nesse cenário, o indivíduo só se dirige a um serviço de saúde quando seu estado já atingiu níveis críticos, devido exatamente ao receio de que possa perder o seu emprego. Em geral, indivíduos de renda mais elevada consultam mais o médico do que os de renda mais baixa. Além disso, existe uma maior proporção de pessoas com plano de saúde nos níveis mais elevados da distribuição de renda. Destaca-se a baixa proporção de pessoas que possuem plano de saúde no setor agrícola, 2,5\%, em relação aos demais, 17,46 (indústria) e 25,92 (comércio e serviços). Tem-se uma evidência clara do risco moral, em que indivíduos com plano de saúde demandam mais serviços médicos do que aqueles que utilizam o Sistema Único de Saúde.

Em contraponto, a proporção de pessoas que esteve acamada nas duas últimas semanas é menor entre aqueles que fazem parte dos decis mais baixos da população. Quanto à utilização de medicamentos contínuos, percebe-se um valor consideravelmente elevado em todos os setores de atividade, mas um comportamento bastante interessante: os indivíduos de maior elevado decil de renda utilizam mais medicamentos de uso contínuo do que aqueles de decis mais baixos. Já quando se analisa a questão de se o medicamento foi fornecido sem

\section{POLÊM!CA $\mid$ LABORE}


custos para os indivíduos, nota-se que as pessoas do setor de indústria e comércio e serviços são as que menos recorreram ao setor público para financiar os seus gastos em medicamentos. Como visto anteriormente, são nesses setores que se encontram a maior proporção de pessoas que possuem plano de saúde, o que está em consonância com os dados referentes à gratuidade dos medicamentos, já que os gastos privados em medicamentos são menores se o indivíduo recorre ao SUS. É válido destacar que as pessoas com nível de renda mais baixo são as que mais receberam medicamentos gratuitos, evidenciando o perfil da demanda de saúde atendida pelo SUS.

Analisando questões socioeconômicas de hábitos, obtém-se algumas conclusões importantes. A proporção de pessoas que praticam algum tipo de exercício físico é menor no setor agrícola e relativamente semelhante nos outros dois setores. Além disso, a prática do esporte é maior entre aqueles que pertencem a decis mais elevados de renda, comportamento contrário quando é analisado o fato do indivíduo fumar. Nesse caso, o setor de comércio e serviços apresenta as menores proporções de fumantes, de modo que as pessoas de níveis de distribuição de renda mais baixo fumam mais do que as de níveis de renda mais elevado. Esse comportamento é comum também aos demais setores de atividade.

Por fim, em relação à renda e à escolaridade (medida em anos de estudo), percebe-se que o setor agrícola apresenta o pior desempenho nesses quesitos. A média de anos de estudos deste setor é cerca de duas vezes menor quando comparada com o setor que apresentou o melhor resultado, comércio e serviços. Além disso, a média da renda de todas as fontes do setor de agrícola é cerca de apenas $45 \%$ da média da renda de todas as fontes do setor de comércio e serviços. Como era de se esperar, nos decis mais baixos da distribuição de renda encontra-se a menor proporção de anos de escolaridade e de renda de todas as fontes.

Tabela - 2: Caracterização de alguns aspectos da saúde controlando por decis de renda e setores de atividades

\begin{tabular}{|c|c|c|c|c|c|c|c|c|c|c|c|}
\hline & \multicolumn{11}{|c|}{ Decis de Renda } \\
\hline \multicolumn{12}{|l|}{ Agricultura } \\
\hline $\begin{array}{l}\text { Considera o Estado de Saúde } \\
\text { Bom ou Muito Bom }\end{array}$ & 56.25 & 59.93 & 64.59 & 67.37 & 56.34 & 56.45 & 56.57 & 61.17 & 50.89 & 60.72 & 59.25 \\
\hline $\begin{array}{l}\text { Esteve acamado nas duas } \\
\text { últimas semanas }\end{array}$ & 42.22 & 34.78 & 43.19 & 66.49 & 32.82 & 55.00 & 28.58 & 42.39 & 73.72 & 62.35 & 46.67 \\
\hline Tem plano de saúde & 1.07 & 1.60 & 3.44 & 0.00 & 2.37 & 0.00 & 2.16 & 2.07 & 4.54 & 27.61 & 2.50 \\
\hline
\end{tabular}

\section{POLÊM!CA $\mid$ LABORE}


Consultou médico nos últimos

12 meses

66.72

55.38

55.86

57.29

71.79

63.27

56.63

Utiliza medicamentos de uso

$18.05 \quad 15.75$

10.15

9.73

22.81

31.72

22.34

15.79

$32.96 \quad 38.22$

19.67

continuo

Este medicam gratuitamente

$60.07 \quad 80.82$

65.59

59.32

61.29

73.77

$45.97 \quad 40.00$

60.12

Praticou algum tipo de

exercício físico ou esporte

$\begin{array}{lll}16.65 & 12.24 \quad 15.44\end{array}$

19.49

22.78

14.29

8.47

10.62

$14.92 \quad 30.95$

15.09

Atualmente, fuma

algum

produto do tabaco

35.87

47.17

13.11

23.53

42.59

35.6

$0.00 \quad 0.00$

27.27

Nas duas últimas semanas,

deixou de realizar quaisquer de

suas atividades habituais por

$13.23 \quad 9.19$

10.23

14.64

10.2

10.75

$10.09 \quad 7.94$

$15.16 \quad 16.35$

11.12

motivo de saúde

Renda de todas as fontes

$\begin{array}{llll}64.67 & 127.33 & 241.70 & 369.00\end{array}$

415.00

415.00

473.40

$627.73 \quad 915.20$

2733.05

400.42

Anos de estudos médio

$\begin{array}{lll}4.11 & 4.21 \quad 3.78\end{array}$

4.04
3.66 3.22 3.20

3.87
3.85 6.97 3.88

\section{Indústria}

Considera o Estado de Saúde

Bom ou Muito Bom

64.

Esteve acamado nas duas últimas semanas

Tem plano de saúde

Consultou médico nos últimos

12 meses

$64.13 \quad 71.13$

74.04

71.01

77.38

83.29

73.32

78.02

76.52

84.91

75.80

$38.52 \quad 43.55$

73.39

$\begin{array}{lll}4.13 & 2.17 \quad 3.68\end{array}$

33.97

78.23

40.00

50.73

38.52

20.46

76.53

50.43

$65.34 \quad 60.10-52.23$

6.70

12.34

10.63

14.92

17.82

33.78

58.80

17.46

Utiliza medicamentos de uso contínuo

65.3460.

52.23

$53.44 \quad 55.11$

54.43

63.83

56.80

63.72

65.78

58.48

$13.02 \quad 7.47$

$12.15 \quad 13.86$

11.61

$13.27 \quad 27.54$

13.25

Este medicamento foi obtido gratuitamente

$51.35 \quad 66.64 \quad 82.66$

$62.28 \quad 70.32$

37.50

53.34

55.87

33.01

19.52

48.82

Praticou algum tipo de exercício físico ou esporte

$27.47 \quad 26.94$

25.46

$24.70 \quad 21.90$

18.23

27.59

22.30

$25.77 \quad 31.25$

25.03

$\begin{array}{lllll}\text { Atualmente, fuma } & \text { algum } & 28.20 & 76.16 & 46.07\end{array}$ produto do tabaco

$30.29 \quad 27.19$

20.00

13.35

12.82

$22.60 \quad 8.39$

25.39

Nas duas últimas semanas, deixou de realizar quaisquer de suas atividades habituais por 10.747 .04 9.36

6.14

6.97

7.59

8.01

6.34

5.27

6.04

7.11 motivo de saúde

Anos de estudos médio

$68.93-142.41$

236.74

387.84

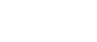

415.01

Comércio e Serviços

Considera o Estado de Saúde

Bom ou Muito Bom

$\begin{array}{lll}6.47 & 5.79 & 6.58\end{array}$
6.97

7.37

6.87

478.57

$\begin{array}{llll}625.66 & 935.23 & 2886.37 & 680.90\end{array}$

$\begin{array}{llllll}7.81 & 8.25 & 9.03 & 11.53 & 7.84\end{array}$

Esteve acamado nas duas últimas semanas

Tem plano de saúde

$\begin{array}{llllllllllll}71.08 & 68.46 & 71.55 & 73.94 & 77.41 & 73.49 & 74.90 & 76.34 & 77.83 & 82.84 & 75.71 \\ 49.79 & 54.11 & 54.64 & 40.75 & 43.78 & 17.52 & 50.34 & 43.09 & 44.99 & 34.33 & 44.66 \\ 7.42 & 6.14 & 7.41 & 10.29 & 16.52 & 12.29 & 19.12 & 26.52 & 41.32 & 67.73 & 25.92\end{array}$

\section{POLÊM!CA $\mid$ LABORE}

Polêmica - Revista Eletrônica da Uerj - Rua São Francisco Xavier, 524, $1^{\circ}$ andar bloco D, sl.1001 • Tels.: +55 21 2334-4088/4087 • http://www.e-publicacoes.uerj.br/index.php/polemica/index http://www.labore.uerj.br • laboreuerj@yahoo.com.br 


\begin{tabular}{|c|c|c|c|c|c|c|c|c|c|c|c|}
\hline $\begin{array}{l}\text { Consultou médico nos últimos } \\
12 \text { meses }\end{array}$ & 61.75 & 67.52 & 63.76 & 64.00 & 64.84 & 62.44 & 65.44 & 67.86 & 68.00 & 73.88 & 66.73 \\
\hline $\begin{array}{l}\text { Utiliza medicamentos de uso } \\
\text { contínuo }\end{array}$ & 13.43 & 18.31 & 16.36 & 11.99 & 14.17 & 15.69 & 16.56 & 15.78 & 20.00 & 27.06 & 17.65 \\
\hline $\begin{array}{l}\text { Este medicamento foi obtido } \\
\text { gratuitamente }\end{array}$ & 71.23 & 69.12 & 71.97 & 59.43 & 50.51 & 60.55 & 54.83 & 41.98 & 36.83 & 13.22 & 44.86 \\
\hline $\begin{array}{l}\text { Praticou algum tipo de } \\
\text { exercício físico ou esporte }\end{array}$ & 22.95 & 18.59 & 19.88 & 19.58 & 22.90 & 20.23 & 25.47 & 29.12 & 29.49 & 37.27 & 25.94 \\
\hline $\begin{array}{l}\text { Atualmente, fuma algum } \\
\text { produto do tabaco }\end{array}$ & 15.92 & 22.67 & 21.30 & 17.01 & 21.93 & 23.81 & 14.09 & 16.42 & 15.69 & 10.48 & 16.59 \\
\hline $\begin{array}{l}\text { Nas duas últimas semanas, } \\
\text { deixou de realizar quaisquer de } \\
\text { suas atividades habituais por } \\
\text { motivo de saúde }\end{array}$ & 9.74 & 10.59 & 7.45 & 8.14 & 5.66 & 5.89 & 7.44 & 5.73 & 6.88 & 5.93 & 7.08 \\
\hline Renda de todas as fontes & 65.70 & 139.26 & 242.92 & 386.61 & 415.00 & 415.02 & 480.79 & 627.00 & 947.19 & 3220.78 & 882.02 \\
\hline Anos de estudos médio & 7.00 & 7.02 & 7.52 & 8.49 & 9.29 & 9.15 & 9.26 & 9.91 & 10.87 & 13.15 & 9.60 \\
\hline
\end{tabular}

Fonte: PNAD (2008), elaboração própria.

\section{Análise dos resultados}

Os resultados do modelo probit sobre a probabilidade do indivíduo possuir determinada doença crônica, com um foco nos setores de atividades, indicam que, dentre as 12 (doze) doenças listadas na PNAD, apenas três variáveis que captam o efeito do setor de atividade mostraram-se significante estatisticamente. Vale ressaltar que os resultados constam na Tabela 3 e indicam o efeito marginal ao invés dos coeficientes estimados do probit ${ }^{3}$. De acordo com a tabela, trabalhar na agricultura aumenta a probabilidade das pessoas possuírem doença de coluna ou costas em relação às demais atividades produtivas. Além disso, quanto maior a escolaridade do indivíduo menor a probabilidade de ter doença de coluna ou costas e, quanto mais velha a pessoa, maior a probabilidade de ser acometida por essa doença. Isso porque quanto mais instruídas são as pessoas, mais acesso à informação elas terão e possivelmente estarão cientes de como se prevenir ou tratar de tal doença.

Em relação à probabilidade do indivíduo ter ou não hipertensão, os resultados da Tabela 3 mostram que trabalhar na agricultura reduz a probabilidade das pessoas possuírem essa doença em comparação ao fato de trabalhar na indústria ou comércio e serviços. No entanto, a variável só foi significativa para explicar o setor terciário. As mulheres apresentam

\footnotetext{
${ }^{3}$ Mais informações ver Wooldridge (2010).
}

\section{POLÊM!CA $\mid$ LABORE}


maior probabilidade de possuírem hipertensão, em relação aos homens e, à medida que envelhecem, maior a probabilidade das pessoas terem essa doença.

Ademais, fazer atividades físicas, possuir um maior nível de escolaridade e morar em área urbana diminuem a probabilidade de o indivíduo possuir hipertensão, apesar desses efeitos marginais serem estatisticamente insignificantes. É interessante notar que, apesar de não apresentar significância estatística, a medida que a renda aumenta maior a probabilidade das pessoas terem hipertensão, hipótese discutida em seções anteriores. Naquela ocasião, argumentou-se que a carga de stress, relacionada com as condições de vida daqueles que pertencem aos mais altos decis de renda, poderia estar indicando uma maior probabilidade de que esses indivíduos tenham hipertensão.

Por fim, o fato das pessoas estarem vinculadas aos setores industriais e comércio e serviços aumenta a probabilidade de serem acometidas por tuberculose, em relação ao setor agrícola (Tabela 3). Esse resultado é semelhante ao verificado no caso da hipertensão. Além disso, possuir um maior nível de escolaridade diminui a probabilidade dos agentes terem tuberculose. Outras variáveis como sexo, idade, cor, área censitária apresentaram o comportamento esperado pela literatura, contudo não foram significantes estatisticamente.

Tabela - 3: Efeitos Marginais para diferentes doenças, Pernambuco, 2008

\begin{tabular}{llll}
\hline Variáveis Independentes & $\begin{array}{l}\text { Doença de Coluna ou } \\
\text { Costas }\end{array}$ & Hipertensão & Tuberculose \\
\hline Sexo & -0.007 & $-0.045^{* *}$ & -0.001 \\
Idade & $(-0.026)$ & $(-0.022)$ & $(-0.022)$ \\
& $0.004 * * *$ & $0.006 * * *$ & 0.000 \\
Cor & $(-0.001)$ & $(-0.001)$ & $(-0.001)$ \\
Urbanização & 0.018 & -0.002 & -0.002 \\
Anos Escolaridade & $(-0.024)$ & $(-0.020)$ & $(-0.020)$ \\
& 0.060 & -0.037 & 0.000 \\
Ln renda de todas as fontes & $(-0.033)$ & $(-0.041)$ & $(-0.041)$ \\
& $-0.008 * *$ & -0.004 & $0.000 *$ \\
Fuma & $(-0.003)$ & $(-0.003)$ & $(-0.003)$ \\
& 0.019 & 0.014 & $0.000^{* * *}$ \\
Atividade Física & $(-0.014)$ & $(-0.012)$ & $(-0.596)$ \\
& -0.005 & -0.026 & dropped
\end{tabular}

\section{POLÊM!CA $\mid$ LABORE}

Polêmica - Revista Eletrônica da Uerj - Rua São Francisco Xavier, 524, $1^{\circ}$ andar 


\begin{tabular}{llll} 
Indústria & $-0.086^{* *}$ & 0.055 & 0.004 \\
& $(-0.033)$ & $(-0.048)$ & $(-0.006)$ \\
Comércio e Serviços & $-0.103^{* *}$ & $0.081^{*}$ & $0.002^{* *}$ \\
& $(-0.044)$ & $(-0.052)$ & $(-0.002)$ \\
\hline
\end{tabular}

Nota: *** indica significância a 1\%; ** indica significância a 5\%; * indica significância a 10\%. Desvio-padrão indicado entre parênteses.

Fonte: PNAD/IBGE 2008, elaboração própria.

\section{Considerações finais}

Este trabalho teve como objetivo realizar uma análise das condições gerais de saúde da população do Estado de Pernambuco, com um olhar no impacto dos setores de atividades em relação às doenças crônicas e aspectos da saúde dos indivíduos. Verificou-se que, em geral, os indivíduos do setor agrícola são os que possuem piores condições de renda e escolaridade e isso acaba impactando de forma negativa nas características de saúde dessa população. Pessoas de renda mais elevada possuem um melhor estado de saúde. Existe, ainda, uma maior proporção de pessoas com plano de saúde nos níveis mais elevados da distribuição de renda, o que influencia a questão do acesso e qualidade dos serviços de saúde.

O SUS, cujo perfil da demanda é caracterizado por pessoas de baixo nível de escolaridade e poder aquisitivo, sofre com problemas de baixa qualidade dos serviços prestados, hospitais lotados, leitos insuficientes e problemas de gestão de toda natureza. Em um mundo à parte, aqueles que estão em um nível de distribuição de renda mais alto parecem que estão alheios a todas essas questões que foram levantadas. Como possuem e pagam caro para ter acesso ao um plano de saúde, utilizam um sistema de saúde distinto daquele descrito acima, em que, por exemplo, tecnologia de ponta é utilizada em tratamentos médicos.

Com uma maior capacidade de organização e vocalização de suas reivindicações, as pessoas pertencentes a estratos sociais elevados conseguem usufruir de um sistema de saúde, se não excelente, bem melhor do que o oferecido pelo governo. Segundo Fahel (2008), essas pessoas encontram respostas razoáveis as suas demandas de saúde na área privada, enquanto os demais (usuários exclusivos do SUS), além da pluralidade e pulverização de interesses, encontram barreiras políticas e sociais para expressão e viabilização das suas reivindicações.

No que se refere à probabilidade do indivíduo possuir determinada doença crônica, com um foco nos setores de atividades, indicam que dentre as 12 (doze) doenças listadas na PNAD apenas três variáveis que captam o efeito do setor de atividade mostraram-se significante estatisticamente. São elas doença de coluna ou costas, hipertensão e tuberculose.

\section{POLÊM!CA | LABORẸ}

Polêmica - Revista Eletrônica da Uerj - Rua São Francisco Xavier, 524, $1^{\circ}$ andar

bloco D, sl.1001 • Tels.: +55 21 2334-4088/4087 • http://www.e-publicacoes.uerj.br/index.php/polemica/index

http://www.labore.uerj.br • laboreuerj@yahoo.com.br 
Além disso, diferenças de gênero e escolaridade apresentaram relativo grau de importância. Isso sugere que políticas públicas na área de saúde devem ser aplicadas concomitantemente a políticas voltadas para mulher e, principalmente, políticas direcionadas para a área de educação.

Em síntese, a democratização e o acesso ao SUS devem ser perseguidos, buscando cumprir o que determina a Constituição Federal (1988). O fortalecimento e aprimoramento do SUS deve ser uma constante na implantação de políticas públicas no Brasil.

\section{Referências}

BARCELLOS, C; QUITÉRIO, L. A. Vigilância ambiental em saúde e sua implantação no Sistema Único de Saúde. Revista Saúde Pública, vol. 40, nº.1, p.170-1771, fev. 2006.

BURNLEY, I.H. Inequalities in the transition of ischaemic heart disease mortality in new south wales, Australia, 1969-1994. Social Science and Medicine, v.47, n.9, p.1209-22, 1998.

GODOY, M. R.; NETO, G. B.; BARROS, P. P.; RIBEIRO, E. P. Estimando as perdas de rendimento devido a doença renal crônica no Brasil. Revista Divulgação em Saúde para Debate, nº.38, p. 68-85, 2007.

KAPLAN, G.A. People and places: contrasting perspectives on the association between social class and health. International Journal of Health Services, v.26, n.3, p. 507-19, 1996.

KAPLAN, G.A.; LYNCH, J.W. Socioeconomic considerations in the primordial prevention of cardiovascular disease. Preventive Medicine, v.29, p. 30-5, 1999.

LACAZ, F. A. C. Qualidade de vida no trabalho e saúde/doença. Ciência e Saúde Coletiva, v. 5, n. 1, 2000. p. $151-161$.

MENDES, R. Conceito de Patologia do Trabalho. In: Patologia do Trabalho. 2 ed. São Paulo: Atheneu, 2005. v. 1. p. 47-92.

MICHAUX, R.; FERRIÈRES, J.; RUIDAVETS, J.B. CAMBOU, J.P.; POUS, J. Statut socio-économique et facteurs de risque coronarien: Etude chez 2.610 hommes âgés de 50 à 60 ans. Santé Publique, v.8, n.4, p. 31527, 1996.

MINAYO, M.C.S. A saúde em estado de choque. Rio de Janeiro: Espaço e Tempo, 1992.

MINAYO, M. C. S.; LACAZ, F. A. C. Saúde do trabalhador: novas-velhas questões. Ciência e Saúde Coletiva, Rio de Janeiro, v. 10, n. 4, p. 797-807, 2005.

MINISTÉRIO DA SAÚDE. Representação no Brasil da OPAS/OMS. Doenças Relacionadas ao Trabalho: manual de procedimentos para serviços de saúde. Brasília: Ministério da Saúde do Brasil, 2001.

OIT. Introdutory report: Decent work - safe work. XVII Congresso n Safety and Fealth at Work, Genebra, 2005.

ROSENGREN, A.; ORTH-GOMER, K.; WILHELMSEN, L. Socioeconomic differences in health indices, social networks and mortality among Swedish men: a study of men born in 1933. Scandinavian Journal of Social Medicine, v. 26, n. 4, p.272-80, 1998.

\section{POLÊM!CA $\mid$ LABORE}


SCHILLING, R. S. F. More effective prevention in occupational healph practice? J Soc Occup Med. 34:71-9; 1984.

SOUZA, N. S. S.; SANTANA, V. S.; OLIVEIRA, P. R. A.; BARBOSA-BRANCO, A. Doenças do trabalho e benefícios previdenciários relacionados à saúde, Bahia, 2000. Revista Saúde Pública, vol. 42, n. 1. p. 630-638, 2008.

WOOLDRIDGE, J. M. Introdução à econometria: uma abordagem moderna. São Paulo: Cengage Learning, 2010.

WUNSCH FILHO, V. Perfil Epidemiológico dos Trabalhadores. Rev. Bras. Med. Trab. Belo Horizonte, vol. 2 , n. 2, p.103-117, 2004.

Recebido em: 25/07/2016.

Aceito em: 30/09/2016.

\section{POLÊM!CA $\mid$ LABORE}

Polêmica - Revista Eletrônica da Uerj - Rua São Francisco Xavier, 524, $1^{\circ}$ andar bloco D, sl.1001 • Tels.: +55 21 2334-4088/4087 • http://www.e-publicacoes.uerj.br/index.php/polemica/index http://www.labore.uerj.br • laboreuerj@yahoo.com.br 